\title{
Observational learning of discriminative avoidance in hooded rats
}

\author{
JON EVAN DEL RUSSO \\ The George Washington University, Washington, D. C. 20005
}

\begin{abstract}
To determine the aspects of an observational situation facilitating subsequent acquisition of an observed task, two experimental groups of rats were exposed to a model's performance of a two-way discriminative shuttlebox avoidance task with a tone as the discriminative stimulus. One experimental group (OND) observed the performance of a naive demonstrator; the other experimental group (OSD) observed a skilled demonstrator. A stimulus control group (SC) was exposed to the tone with no model present; a response control group (RC) observed demonstrator running with no tone present. A naive control group (NC) received no observational experience. All groups were subsequently tested on acquisition of the task. An analysis of variance on avoidance scores and Newman-Keuls tests for differences between means revealed that OND and OSD groups performed significantly more avoidances than the control groups. Exposure to the total tone-running sequence was found to be necessary for observational learning. The results were interpreted in terms of a sensory-sensory conditioning model.
\end{abstract}

The observational learning procedure was designed to determine whether learning can occur through mere exposure to a stimulus sequence, that is, in the absence of performance of the response to be learned and response-contingent reinforcement. In the typical observational learning situation, an experimental subject is allowed to observe the performance of a task by a demonstrator or model of the same species. While the subject is observing, he has no chance to perform the observed behavior and receives no direct reinforcement. After observation, the model is removed from the experimental situation, and the observer is tested on the observed task. If the observer learns the task faster than control subjects that have not observed a model, it is assumed that the observer learned something about the task during observation that facilitated subsequent acquisition.

Several studies (Berry, 1906, 1908; Haggerty, 1909; Thorndike, 1911; Watson, 1908) were carried out in the early part of this century to determine if animals can learn by observation. Positive and negative results were reported, and the issue remained unresolved. This discrepancy in results was probably due to the poor experimental methods and lack of proper control groups that characterized these early experiments. Warden and Jackson (1935), in testing observation learning in monkeys, set up strict criteria for observational learning which eliminated several variables, such as the possibility of trial and error learning, that had contaminated earlier experiments. Warden and Jackson tested rhesus monkeys on observational learning of four tasks: pulling a chain, manipulating a knob, operating a latch, and operating a double latch. Correct performance opened a door, revealing food. The experimenters found that observers performed significantly better than control subjects that did not observe. Several observers performed the

The author is now at Monmouth College, West Long Branch New Jersey 07764. observed task within $10 \mathrm{sec}$, indicating that their behavior was largely determined by observational leaming rather than by trial and error learning. Warden and Jackson concluded that monkeys can learn by observation and do exhibit a strong tendency to imitate.

Since Warden and Jackson's experiment, observational learning has been demonstrated in a number of species and in a variety of learning situations (Hayes \& Hayes, 1952; Herbert \& Harsh, 1944; Myers, 1970). More recently, experimenters have turned their attention from mere demonstration to a closer scrutiny of the observational learning process itself, Adler (1955) conducted an observational learning experiment with cats, testing whether the basic factor involved in observational learning is the locus of demonstrator response or the form of the model's response to the manipulandum. He found that the form of the model's response more significantly affects success on the task than the locus of demonstrator response, although both were found to be significant factors in observational learning. Groesbeck and Duerfeldt (1971) have attempted to examine the relative contribution to observational learning of informational, modeling, following, and vicarious reinforcement components present in the observational situation. Their results indicated that all of the experimental treatments used facilitated the acquisition of the discrimination. Performance of the task by the demonstrator appeared to be the most important aspect of the observational learning situation, with experimental groups that viewed a demonstrator's performance of the discrimination task learning sooner than experimental groups that did not.

One important variable which Groesbeck and Duerfeldt tried to isolate that has not been adequately controlled for in observational learning experiments is the possibility of vicarious reinforcement to observers. Mowrer (1960) claims that mere observation of a model receiving reward is reinforcing to observers. Mowrer 
hypothesizes that the observer vicariously experiences the sensory consequences of the model's behavior. anticipates that a similar reinforcing outcome will result from his own performance of the observed act, and is thus predisposed to repeat the model's behavior. In several studies employing an appetitive situation (Adler, 1955; Herbert \& Harsh, 1944; Warden \& Jackson, 1935), observers have had the opportunity to see the model reinforced during demonstration trials. All of these experiments leave open the possibility that vicarious reinforcement may have facilitated learning by observers. In the present experiment, the effect of a possible source of vicarious reinforcement in the observational learning process is tested.

Presley and Riopelle (1959) and John, Chesler. Bartlett, and Victor (1968) have demonstrated observational learning of discriminative avoidance tasks. Presley and Riopelle allowed observer monkeys to observe naive demonstrators learning to avoid shock by hurdle-jumping. A red light served as the warning stimulus. Presley and Riopelle report that all observers learned faster than the traditionally trained demonstrators, the slowest observer having learned the response in fewer trials than the fastest demonstrator. John et al. had cats observe the performance of a naive demonstrator on a discriminative hurdle-jump response for 20 trials, then the performance of a trained demonstrator for 20 trials. Prior to observation, observers received one test trial on the discriminative hurdle-jump task, referred to as an "empathy" trial by John et al. This procedure was repeated daily until the naive demonstrator reached criterion. Observers were then tested. Five out of six observers learned the response in significantly fewer trials than their paired student demonstrators.

The experiments conducted by John et al. and Presley and Riopelle could have yielded further information about the observational learning process if more stringent controls had been employed. Their results suggest that observers may have learned the cue function of the discriminative stimulus during observation. However, whether observers were merely learning the correct response, or whether the cue function of the stimulus was also being learned during observation is not clear from the results of these experiments. In the present experiment, which also employs discriminative avoidance as an observational learning task, control groups are used which make it possible to separate response learning effects from cue learning effects in the discriminative avoidance situation. If control subjects exposed only to the responise component perform as well as observers exposed to the stimulus-response sequence, observational learning would appear to be primarily a response-learning effect. However, if observers exposed to the total stimulus-response sequence perform significantly better than the response control group, one would conclude that observers had learned the relationship between the warning stimulus and the response (i.e.. the cue function of the warning stimulus) during observation.

Several observational learning studies have not controlled for important variables which may influence the observational learning process. The shortcomings of these earlier studies can be avoided by setting up reasonable criteria for observational learning studies. The following criteria appear to be necessary for a well-designed observational learning experiment: (1) Apparatus criteria. (a) the observation box should be located in such a position that a full view of the test chamber is available to the observer; (b) the observation box should be of a size that will minimize movement by the observer and maximize observation. (2) Response criteria. (a) the response should be one that can be repeated a number of times in succession; (b) the response should not involve intricate or detailed movements which might be difficult to observe. (3) Design criteria. (a) number of observation trials should be equal across observers; (b) possible sources of reinforcement, either direct or vicarious (e.g., delivery of food to model; cessation of model's squeals), should be eliminated or controlled for.

The discriminative avoidance situation used in the present experiment meets the above criteria. The avoidance response can be repeated any number of times in succession and involves running, a response free from intricate movements which might be unobservable. The avoidance situation is especially convenient in controlling for sources of reinforcement. In appetitive situations, the observer is exposed to eating behavior by the model, while in an avoidance situation, a trained model merely performs a response when the warning stimulus is presented, and no visible reinforcement is involved. If the model is well-trained, no squealing or other signs of distress will occur, since shock is avoided.

\section{METHOD}

\section{Subjects}

Fifty Long-Evans hooded rats ranging between 70 and 200 days of age were randomly assigned to five groups. All subjects were individually housed for the duration of the experiment Several other rats of the same species were available for use as demonstrators.

\section{Apparatus}

Apparatus consisted of a shuttlebox and a small observation chamber. The shuttlebox, 29 in. long $\times 11$ in. wide $\times 17$ in. high, was constructed of wood sides and a Plexiglas top. The shut tlebox was divided into two compartments of equal size, one black and one white, by a Plexiglas partition extending from floor to ceiling and from side to side. In the center of the partition was a 3 in. wide $x 4$ in. high door, the bottom of which rested on the floor of the shuttlebox. A wire, strung through the top of the door and run up through a hole in the Plexiglas top, could be used by the experimenter to open the door. The door was covered on both sides with alternating black and white strips 
of tape. The floor of the shuttlebox was a grid consisting of 36 steel bars $1 / 5$ in. in diam, set 3/5 in. apart. Scrambled shock could be delivered to the grid via a model H.I. 3121 shock generator.

A hole 9 in. long $\times 8$ in. high, centered at the Plexiglas partition, was cut in one side of the shuttlebox to allow a view of the inside of the shut tlebox from a 9 in. long $x 8$ in. high $x$ 4 in. wide observation chamber. One side of the metal observation chamber was Plexiglas and was flush with the wall of the shuttlebox.

A speaker which emitted an 8,000 cps tone was mounted on top of the shuttlebox. Stimulus presentations and time intervals were programmed using standard electro-mechanical relay equipment.

\section{Procedure}

Discriminative two-way shuttlebox avoidance was used as an observational learning task. An 8,000 cps tone served as the warning stimulus. Shock could be delivered independently to the left or right side of the shuttlebox on alternate trials.

Each trial began at tone onset, which occurred simultaneously with raising of the guillotine door by the experimenter. Five seconds later shock was delivered. The tone was terminated by the experimenter when a successful avoidance or escape response occurred. The next trial was programmed to begin $20 \mathrm{sec}$ after tone offset.

Before testing was begun, each subject was placed in the observation chamber and was exposed to one of the following observational conditions:

Observers of skilled demonstrator (OSD). While each subject was in the observation box, a skilled demonstrator was placed in the shut tlebox and performed the discriminative avoidance task for 100 trials.

Observers of naive demonstrator (OND). While each subject was in the observation chamber, a naive demonstrator was placed in the shuttlebox and run for 100 trials on the discriminative avoidance task. Naive demonstrators learned the avoidance task while observers watched.

Stimulus control group (SC). While stimulus control subjects were in the observation chamber, the tone was presented 100 times (no demonstrator was present in the shuttlebox).

Response control group (RC). While response control subjects were in the observation chamber, a demonstrator performed 100 avoidance responses with no tone present. Raising of the guillotine door served as the warning stimulus for the RC demonstrator.

Naive control group (NC). Naive control subjects spent a 30-min adaptation period in the observation chamber, but were not exposed to any other aspect of the test situation.

Immediately following the preliminary observation box treatment, all subjects were transferred to the shuttlebox and given 100 test trials on the discriminative avoidance task in an uninterrupted session lasting approximately $1 \mathrm{~h}$.

Several aspects of this observational situation might result in savings to acquisition of the observed task by experimental subjects. Both experimental groups are exposed to information about the form of the demonstrator's response, and information about the cue function of the warning stimulus, i.e., the temporal relationship between the warning stimulus and the demonstrator's response. In addition, experimental subjects that observe a naive demonstrator are exposed to a possible source of vicarious reinforcement. The naive model's initial reactions to shock (squealing, jumping, etc.) and the cessation of these activities with acquisition of the discriminative avoidance task by the model provides a potential source of vicarious reinforcement to these observers. Finally, mere familiarization with the warning stimulus could result in some savings by experimental subjects.

The three control groups were used to determine which of these aspects of the observed situation facilitate subsequent acquisition of the task by observers. The response control group,
Table 1

Group Means and Standard Deviations for Total Avoidances in 100 Trials

\begin{tabular}{lccccc} 
& & \multicolumn{3}{c}{ Group } \\
& OSD & OND & RC & SC & NC \\
\hline Mean & 49.3 & 52.2 & 35.9 & 27.0 & 25.1 \\
SD & 10.6 & 15.8 & 15.5 & 14.5 & 15.8 \\
\hline
\end{tabular}

which observes a model perform avoidance responses with no warning tone present, makes it possible to determine whether mere exposure to the form of the model's response facilitates learning of the discriminative avoidance task by observers. The response control group also controls for the possible reinforcing effect of the mere sight of another active rat. The stimulus control group, which is exposed to presentations of the warning stimulus with no demonstrator present, controls for the effects of familiarization with the warning stimulus. The performance of the two experimental groups and the stimulus and response control groups can be compared to the performance of the naive control group to determine which, if any, of these treatments facilitate subsequent acquisition of the task.

\section{RESULTS}

The mean number of avoidances per group over 100 trials and the standard deviation for each group are presented in Table 1.

An analysis of variance comparing the number of avoidances performed over 100 trials indicated an overall difference between groups $(F=7.26 ; d f=4,45$; $\mathrm{p}<.001$ ). A Newman-Keuls test for differences between group means revealed that observers of naive demonstrators and observers of skilled demonstrators performed significantly more avoidances than stimulus control and naive control groups $(\mathrm{p}<.01)$, and significantly more avoidance than the response control group $(\mathrm{p}<.05)$.

The learning curves for the five groups were plotted by totalling the number of avoidances for each group over blocks of 10 trials, and are presented in Figure 1. A two-way (treatments by blocks) analysis of variance with repeated measures on blocks was done to determine whether or not the rates of learning for the groups were significantly different. The Blocks by Treatments interaction was significant $(F=2.49 ; \quad d f=36,405$; $\mathrm{p}<.01$ ), indicating that the learning rates for the five groups were different. Tests for simple effects revealed which blocks contained significant treatment effects. For those blocks found to contain significant treatment effects. Newman-Keuls tests for differences between means were carried out to determine which groups were significantly different. Table 2 summarizes the results of tests for simple effects and Newman-Keuls tests.

No overall significant differences were found for Blocks 1, 2, and 9. In Block 3, the observers of naive demonstrators performed significantly better than the naive control and stimulus control groups $(p<.05)$. In Blocks 8 and 10 , the observers of naive demonstrators performed significantly better than the naive control 


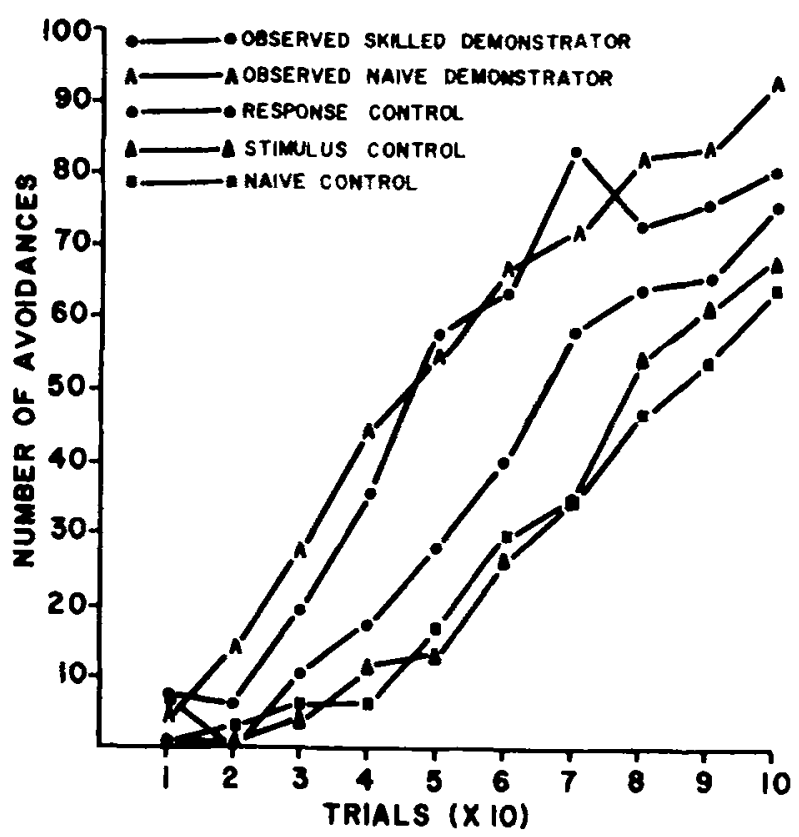

Figure 1. Total number of avoidance responses per group within each block of 10 trials.

group $(p<.05)$. In Blocks $4,5,6$, and 7 the two experimental groups performed rignificantly better than both the stimulus control and naive control groups $(p<.05)$. The two experimental groups performed significantly better than the response control group in Blocks 4 and $5(\mathrm{p}<.05)$.

The analysis of variance on total avoidance scores indicates that observation of the sequence of tone running did result in observational learning, whereas exposure to the tone alone or to the running response alone did not significantly affect subsequent acquisition of the discriminative avoidance task. The analysis of the learning curves reveals that the effect of observation was most apparent in Blocks 4-7. There were no differences between the two experimental groups throughout the 10 blocks, indicating that the potential source of vicarious

Table 2

Summary of Tests on Simple Effects (Treatments at Blocks) and Corresponding Newman-Keuls Tests

\begin{tabular}{|c|c|c|}
\hline Block & $\begin{array}{l}\text { Analysis of Variance } \\
\text { (F) } \mathrm{df}=4 / 45\end{array}$ & Newman-Keuls Tests \\
\hline 1 & 1.54 & \\
\hline 2 & 2.54 & \\
\hline 3 & $3.30^{*}$ & $\mathrm{OND}>\mathrm{SC}=\mathrm{NC}^{*}$ \\
\hline 4 & $6.05^{* *}$ & $\mathrm{OSD}=\mathrm{OND}>\mathrm{RC}=\mathrm{NC}=\mathrm{SC}^{*}$ \\
\hline 5 & $8.66^{* *}$ & $\mathrm{OSD}=\mathrm{OND}>\mathrm{RC}=\mathrm{NC}=\mathrm{SC}^{*}$ \\
\hline 6 & $4.16^{* *}$ & $\mathrm{OSD}=\mathrm{OND}>\mathrm{SC}=\mathrm{NC}^{*}$ \\
\hline 7 & $7.42 * *$ & $\mathrm{OSD}=\mathrm{OND}>\mathrm{SC}=\mathrm{NC}^{*}$ \\
\hline 8 & $3.31^{*}$ & $\mathrm{OND}>\mathrm{NC}$ \\
\hline 9 & 2.08 & \\
\hline 10 & $2.72 *$ & OND $>\mathrm{NC}^{*}$ \\
\hline
\end{tabular}

${ }^{*} p<.05 \quad{ }^{* *} p<.01$ reinforcement for observers of naive demonstrators had no effect on observational learning.

\section{DISCUSSION}

The results demonstrate that rats are capable of observational learning of a discriminative avoidance task. Both experimental groups performed significantly more avoidances in the 100 test trials than did any of the three control groups.

Since neither the stimulus control group nor the response control group performed any differently from naive control animals, it is concluded that neither familiarization with the waming stimulus nor exposure to the running response alone significantly affected learning of the task. The failure of the response control group to demonstrate observational learning indicates that observational learning by experimental subjects was not simply a response learning effect.

Since all possible sources of vicarious reinforcement to observers of skilled demonstrators were controlled for in this experiment, the observational learning demonstrated by the OSD group seems to indicate that reinforcement, either direct or vicarious, is not necessary for observational learning.

An analysis of the learning curves yielded valuable information about the nature of the observational learning which occurred. More group differences were found in Blocks 4, 5, 6, and 7 than in the other blocks. The two experimental groups were superior to the stimulus control and naive control groups in these four blocks, and superior to the response control group in Blocks 4 and 5. It was noted that most subjects demonstrated the same typical learning pattern when placed in the test situation. During the first 15 to 30 trials, subjects established a rapid escape response and learned the correct response of running through the door. After this response learning had occurred, the running response gradually came under the control of the warning stimulus. During this second phase of the learning process (Blocks 4, 5, 6, and 7), the two experimental groups displayed significantly faster acquisition of the discriminative avoidance task than the three control groups. These results indicate that the learning taking place during observation made it easier for observers to establish the connection between the stimulus (tone) and the response (running through the door) during acquisition. Since the only groups that showed observational learning were the two experimental groups that observed the tone-running sequence, it appears that during observation observers exposed to the tone-running sequence learned the connection or association between the discriminative stimulus and the running response of the demonstrator.

One possible explanation of how this type of learning could come about has been suggested by Bandura 
(1969). Bandura claims that during observation a sequence of observed sensory events becomes integrated through a process of sensory-sensory conditioning. When an observer is exposed to the repeated pairing of sensory events, a stimulus component of the observed sequence acquires the capacity to elicit in the observer centrally aroused perceptions of the associated sensory events. The operation of such a sensory-sensory conditioning mechanism might account for the results of the present experiment. Through repeated exposure to the stimulus-response sequence of tone-running, a sensory-sensory connection between the tone and running behavior could have been established for experimental subjects. Subsequent presentation of the tone during acquisition testing would elicit centrally aroused perceptions of the associated running response, and thus facilitate formation of the stimulus-response connection during acquisition testing.

The poorer performance of the response and stimulus control groups can also be explained in terms of the sensory-sensory contiguity conditioning hypothesis. The response control group had no exposure to the tone and thus had no opportunity to learn the tone-running sequence. Similarly, stimulus control subjects were exposed to the tone but not to running behavior, and thus did not observe the complete tone-running sequence. Since both observers of skilled demonstrators and observers of naive demonstrators were exposed to the tone-running sequence, they both had the opportunity to learn the association between the warning stimulus and running behavior.

The sensory-sensory conditioning hypothesis, however, leaves unanswered the question of how sensory-sensory conditioning during observation could transfer to and facilitate stimulus-response learning during acquisition testing. Hebb (1960) has suggested that sight of another performing a particular action tends to produce in an observer the corresponding somesthetic sensations and central processes that would be aroused if the observer performed the response himself. A central mediation process of this type might account for the transfer effect obtained in the present experiment.

\section{REFERENCES}

Adler, H. E. Some factors of observational learning in cats. Journal of Genetic Psychology, 1955, 86, 159-177.

Bandura, A. Principles of behavior modification Rinehart, \& Winston, 1969.

Berry, C. S. The imitative tendency of white rats. Journal of Comparative Neurology and Psychology, 1906, 16, 333-361.

Berry, C. S. An experimental study of imitation in cats. Journal of Comparative Neurology and Psychology, 1908, 18, 1-25.

Groesbeck, R. W., \& Duerfeldt, P. H. Some relevant variables in observational learning of the rat. Psychonomic Science, 1971, 22, 41-43.

Haggerty, M. E. Imitation in monkeys. Century Magazine, 1909 , $78,81-95$.

Hayes, K. J., \& Hayes, C. Imitation in a home-raised chimpanzee. Journal of Comparative and Physiological Psychology, 1952, 45, 450-459.

Hebb, D. O. The American revolution. American Psychologist, $1960,15,735-745$.

Herbert, M. J., \& Harsh, C. M. Observation learning by cats. Journal of Comparative Psychology, 1944, 37, 81-95.

John, E. R., Chesler, P., Bartlett, F., \& Victor, I. Observation learning in cats. Science, 1968, 159, 1489-1491.

Mowrer, $\mathrm{O}$. H. Learning theory and the symbolic processes. New York: Ronald Press, 1960.

Myers, W. A. Observational learning in monkeys. Journal of the Experimental Analysis of Behavior, 1970, 14, 225-235.

Presley, W. J., \& Riopelle, A. J. Observational learning of an avoidance response. Journal of Genetic Psychology, 1959, 95, 251-254.

Thorndike, E. L. Animal intelligence. (1911). New York: Hafner Publishing Co., 1965 (Reprint).

Warden, G. J., \& Jackson, T. A. Imitative behavior in the rhesus monkey. Journal of Comparative Psychology, 1935, 27, 133-147.

Watson, J. B. Imitation in monkeys. Psychological Bulletin, $1908,5,169-178$.

(Received for publication June 24, 1974; revision accepted September $30,1974$. ) 